American Journal of Animal and Veterinary Sciences 5 (3): 208-214, 2010

ISSN 1557-4555

(C) 2010 Science Publications

\title{
Characterization of Escherichia coli Isolates from Commercial Layer Hens with Salpingitis
}

\author{
Mahmood Salehi and Reza Ghanbarpour \\ Department of Poultry Diseases and Microbiology, Faculty of Veterinary Medicine, \\ Shahid Bahonar University, P.O. Box 76169-14111, Kerman, Iran
}

\begin{abstract}
Problem statement: Escherichia coli isolates are the most common bacterial agents of salpingitis in commercial layer hens. The objectives of this study were to determine the phylogenetic groups/subgroups and antibiotic resistance of $E$. coli isolates from salpingitis cases in commercial layer hens farms in southeast of Iran. Approach: One hundred twenty one E. coli isolates from oviducts of layer hens with salpingitis were examined to determine their O-serogroup, phylogenetic group/subgroup and antibiotic resistance pattern. Results: O-serogroup determination test showed that $47(38.84 \%)$ isolates were typeable and belonged to eleven different $\mathrm{O}$ serogroups including: $\mathrm{O} 1, \mathrm{O} 2$, O6, O8, O15, O20, O25, O36, O78, O86 and $\mathrm{O} 111$ and $71(58.67 \%)$ isolates were O-nontypeable. Three most prevalent serogroup were O78, $\mathrm{O} 2$ and $\mathrm{O} 1$ respectively. PCR assays showed that the isolates fall into four phylogenetic groups A (41.32\%), D (33.88\%), B2 (14.87\%) and B1 (9.91\%). Ninety nine $(81.81 \%)$ isolates fell into six phylogenetic subgroups including: $\mathrm{A}_{0}(17.35 \%), \mathrm{A}_{1}$ (23.96\%), B2 $2(5.78 \%), B 2_{3}(9.09 \%), \mathrm{D}_{1}(13.22 \%)$ and $\mathrm{D}_{2}(20.66 \%)$. All of the $E$. coli isolates from $\mathrm{O} 78$ serogroup belonged to A phylo-group whereas the $\mathrm{O} 2$ isolates mostly fell into $\mathrm{D}$ group. The maximum antibiotic resistance was against tetracycline $(100 \%)$ and minimum resistance was against linco-spectin (37.19\%). Twenty four isolates (19.83\%) were resistant to all of the examined antibiotics. Twenty three different patterns of multiple drug resistance were observed, out of which N-Te-Sxt-FmNfx-C and N-Te-Sxt-Gm-Fm-Nfx-C-Ls was the two most frequent patterns respectively. The resistant isolates were found in all of the phylogenetic groups and or sub groups. Conclusion: There are similarities between salpingitis derived E. coli strains and other avian pathogenic $E$. coli isolates in phylogroups, O-serogroups and antibio-resistance patterns.
\end{abstract}

Key words: Escherichia coli, antibiotic, phylotyping, salpingitis

\section{INTRODUCTION}

Salpingitis is an inflammation of the oviduct. Spread of bacteria into the body cavity through the compromised oviduct wall leads to concurrent peritonitis, which is termed salpingoperitonitis (Saif et al., 2003). Environmental stress has been shown to be a factor that may induce colonization of food animals by enteric pathogens, facilitate horizontal transmission of pathogens between animals, increase pathogen shedding (Soliman et al., 2009). Salpingitis a complex condition of chickens associated with various bacterial agents including Escherichia coli (E. coli), Gallibacterium anatis, Staphylococcus spp., Mannheimia haemolytica, Streptococcus bovis and occasionally Salmonella spp. (Jordan et al., 2005; Neubauer et al., 2009). E. coli isolates are the most common bacteria which have been reported from many countries as a frequent cause of disease in commercial laying hens (Fossum et al., 2009). On the other hand $E$. coli is one of the opportunist pathogen responsible for number of disease conditions such as yolk sac infection, air sac disease, perihepatitis, enteritis, omphalitis, coligranuloma and colibacillosis (Ghanbarpour and Salehi, 2010). Avian Pathogenic E. Coli (APEC) serovars involved as causative agents of salpingitis in all birds (layers and broiler breeders, turkeys, ducks and geese) that are sexually mature (Pattison et al., 2007). APEC strains belong predominantly to serogroups O1, O2, O5, O8, $\mathrm{O} 18$ and $\mathrm{O} 78$ (Germon et al., 2005). The method of spread to the reproductive tract is unknown. Several routes of infection have been described: ascending fecal contamination from the cloaca, bacterial translocation respiratory tract (air sac and

Corresponding Author: Mahmood Salehi, Department of Poultry Diseases, Faculty of Veterinary Medicine, Shahid Bahonar University of Kerman, P.O. Box 7616914111, Kerman, Iran Tel: (98) 3412123709 Fax: (98) 3413222047 
lungs) and bacterial translocation from the intestinal lumen (Landman and Cornelissen, 2006). However, the pathogenesis of E. coli isolates salpingitis and peritonitis has not been completely elucidated yet. According to the serogroups involved, pathogenicity for day-old chicks and virulence indicators, the salpingitis isolates were similar to those from cases of chronic respiratory disease (Monroy et al., 2005).

Currently, there are four well-recognized phylogroups of $E$. coli strains and these have been designated A, B1, B2 and D. Genome size varies among the four phylo-groups with A and B1 strains having smaller genomes than B2 or D strains (Gordon et al., 2008). However, to increase the discriminative power of phylogenetic analyses, E. coli strains have been segregated in seven phylogenetic subgroups $\left(\mathrm{A}_{0}, \mathrm{~A}_{1}\right.$, $\mathrm{B} 1, \mathrm{~B} 2_{2}, \mathrm{~B} 2_{3}, \mathrm{D}_{1}, \mathrm{D}_{2}$ ) according to presence/absence of three genetic markers chuA, yjaA and Tspe4.C2 (Escobar-Paramo et al., 2004b). E. coli isolates from main phylo-groups appear to differ in their ecological niches, life-history characteristics and propensity to cause disease (Walk et al., 2007). The extra-intestinal pathogenic strains belong mainly to phylogenetic groups B2 and, at a lesser extend, D and possess numerous specific virulence genes that are absent in commensal strains (Escobar-Paramo et al., 2006). Strains of the phylo-groups also differ in their phenotypic characteristics, such as antibiotic-resistance profiles, ability to exploit different sugars and growth rate-temperature relationships (Escobar-Paramo et al., 2004a; Gordon et al., 2005).

Currently APEC infections in poultry are controlled indirectly by protecting against primary respiratory pathogens. In many countries it has been common practice to administer antibiotics to suppress infection with APEC. Antimicrobials, commonly administered as feed supplements, are fundamental to treating these conditions but their persistent, frequent administration can create multi-antimicrobial resistant bacteria (Johnson et al., 2003; Li et al., 2010).

The objectives of this study were to determine the phylogenetic groups/subgroups and antibiotic resistance of $E$. coli isolates from salpingitis cases in commercial layer hens farms in southeast of Iran.

\section{MATERIALS AND METHODS}

Bacterial strains: A total of 121 E. coli isolates were obtained from oviducts of layer hens with salpingitis and/or salpingoperitonitis. Isolates were collected between 2007 and 2008 from different farms and flocks in Kerman province (south-eastern), Iran. The sampled birds were aged between 30 and 68 weeks. Standard biochemical and bacteriological methods were used to isolate and identify the E. coli strains.

Several strains from the ECOR collection were used as controls for phylogenetic grouping: ECOR58 (B1 group), ECOR50 (D group) and ECOR62 (B2 group). E. coli strain MG1655 was used as a negative control for virulence typing and as a positive control for phylogenetic ECOR group A. The reference strains were a kind gift from Eric Oswald (Ecole National Veterinaire Toulouse, France). All the bacterial strains were routinely grown in Luria-Bertani broth at $37^{\circ} \mathrm{C}$ with aeration and stored at $-70^{\circ} \mathrm{C}$ in $30 \%$ sterile glycerol until they were used. For preparation of samples for testing, isolates were cultured on Trypticase-Soy Agar (TSA) and/or Trypticase-Soy Broth (TSB) at $37^{\circ} \mathrm{C}$ over night.

Serogroup determination and Phylotyping assay: $O$ serogroup of $E$. coli isolates were examined by using specific antisera provided from LREC, University of Santiago de Compostela, Lugo, Spain.

Freshly grown over night cultures of E. coli isolates and reference strains were used for DNA extraction by boiling. The triplex PCR method was used to assign the E. coli isolates (Clermont et al., 2000). The specific primers (TAG Copenhagen, Denmark) used for amplification of the genes. The Clermont method has the potential to yield seven distinct phylogenetic groups and subgroups $\left(\mathrm{A}_{0}, \mathrm{~A}_{1}, \mathrm{~B} 1\right.$, $\mathrm{B} 2_{2}, \mathrm{~B} 2_{3}, \mathrm{D}_{1}$ and $\left.\mathrm{D}_{2}\right)$. The phylo-type of each isolate was determined as follows; chu $\mathrm{A}^{-}, \mathrm{Yja} \mathrm{A}^{-}$, TSPE4 $\mathrm{C}^{-}$ was assigned to subgroup $\mathrm{A}_{0}$ (group $\mathrm{A}$ ), $\mathrm{Chu} \mathrm{A}^{-}, \mathrm{Yja}$ $\mathrm{A}^{+}$, TSPE4.C2 ${ }^{-}$was assigned to subgroup $\mathrm{A}_{1}$ (group $\mathrm{A}$ ); Chu $\mathrm{A}^{-}, \mathrm{Yja} \mathrm{A}^{-}$, TSPE4C2 $2^{+}$was assigned to group $\mathrm{B} 1$, $\mathrm{Chu} \mathrm{A}^{+}, \mathrm{Yja}^{+}$, TSPE4C2 ${ }^{-}$was assigned to subgroup $\mathrm{B} 2{ }_{2}$ (group B2), Chu $\mathrm{A}^{+}$, Yja $\mathrm{A}^{+}$, TSPE4C2 ${ }^{+}$was assigned to subgroup $\mathrm{B}_{3}$ (group B2), Chu $\mathrm{A}^{+}, \mathrm{Yja} \mathrm{A}^{-}$, TSPE4C2 2 was assigned to subgroup $\mathrm{D}_{1}$ (group $\mathrm{D}$ ) and

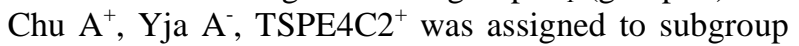
$\mathrm{D}_{2}$ (group D) (Escobar-Paramo et al., 2004a; 2004b) Isolates failing to produce any PCR products were repeated twice more using newly prepared DNA extract. After 2-3 attempts, any isolate negative for all three PCR products was confirmed to be E. coli by using biochemical API 20E identification kit (BioMe'rieux, Marcy l'Etoile, France). If the isolate was identified as E. coli, it was considered to be from subgroup $\mathrm{A}_{0}$ (phylogroup A).

Antibiotic susceptibility test: Resistances against to eight antibiotics were determined by disc diffusion method according to Clinical and Laboratory Standards Institute's (CLSI) guidelines (Jorgensen and Hindler, 
2007). The following antimicrobial discs (Padtan-Teb, Tehran, Iran) were used for all isolates: Chloramphenicol (C), enrofloxacin (Nfx), Flumequine (Fm), Gentamycin (Gm), linco-spectin (Ls), Neomycin (N), oxytetracycline (Te) and trimethoprimsulfamethxazole (Sxt).

\section{RESUTLS}

O-serogroup determination test showed that 47 $(38.84 \%)$ isolates were typeable and belonged to eleven different $\mathrm{O}$ serogroups including: $\mathrm{O} 1, \mathrm{O} 2, \mathrm{O} 6, \mathrm{O} 8, \mathrm{O} 15$, O20, O25, O36, O78, O86 and $\mathrm{O} 111$ and 71 (58.67\%) isolates were O-nontypeable. Nineteen isolates (15.70\%) belonged to O78 serogroup, which was the most prevalent followed by $\mathrm{O} 2(8.26 \%)$ and $\mathrm{O} 1$ (4.95\%) serogroups (Table 1$)$.

PCR assays revealed that $121 \mathrm{E}$. coli isolates fall into four phylogenetic groups whereas $41.32 \% \quad(50$ isolates) belonged to A, $33.88 \%$ (41) to $\mathrm{D}, 14.87 \%$ (18) to $\mathrm{B} 2$ and $9.91 \%$ (12) to B1 groups (Table 1).

Table 1: O-serogroups of 121 E. coli isolates from salpingitis of hens in relation to phylo-groups

\begin{tabular}{|c|c|c|c|c|c|}
\hline \multirow[b]{2}{*}{ O-group } & \multicolumn{4}{|c|}{ Phylogenetic group } & \multirow[b]{2}{*}{ Total No. (\%) } \\
\hline & A & B1 & B2 & $\mathrm{D}$ & \\
\hline O1 & - & - & 4 & 2 & $6(4.95)$ \\
\hline $\mathrm{O} 2$ & - & - & 3 & 7 & $10(8.26)$ \\
\hline O6 & - & - & 1 & - & $1(0.82)$ \\
\hline $\mathrm{O} 8$ & - & - & & 1 & $1(0.82)$ \\
\hline $\mathrm{O} 15$ & - & 1 & - & - & $1(0.82)$ \\
\hline $\mathrm{O} 20$ & - & - & - & 2 & $2(1.65)$ \\
\hline $\mathrm{O} 25$ & - & - & - & 1 & $1(0.82)$ \\
\hline O36 & 2 & - & - & - & $2(1.65)$ \\
\hline $\mathrm{O} 78$ & 19 & - & - & - & $19(15.70)$ \\
\hline O86 & - & 1 & - & - & $1(0.82)$ \\
\hline O111 & - & - & - & 3 & $3(2.47)$ \\
\hline $\mathrm{NT}^{*}$ & 14 & 4 & 4 & 10 & $74(61.15)$ \\
\hline Total & $50(41.32 \%)$ & $12(9.91 \%)$ & $18(14.87 \%)$ & $41(33.88 \%)$ & $121(100)$ \\
\hline
\end{tabular}

*: Nontypeable

Table 2: Multiple antibiotic resistance patterns and phylogenetic groups and/or subgroups of $121 \mathrm{E}$. coli isolates from hens with salpingitis

\begin{tabular}{|c|c|c|c|c|c|c|c|c|}
\hline \multirow[b]{2}{*}{ Resistance pattern } & \multicolumn{6}{|c|}{ Phylo-group/ subgroup } & \multirow[b]{2}{*}{$\mathrm{D}_{2}$} & \multirow[b]{2}{*}{ Total No. } \\
\hline & $\mathrm{A}_{0}$ & $\mathrm{~A}_{1}$ & B1 & $\mathrm{B} 2_{2}$ & $\mathrm{~B} 22_{3}$ & $\mathrm{D}_{1}$ & & \\
\hline N Te Sxt Gm Fm Nfx C Ls* & 4 & 7 & 2 & 3 & 1 & 1 & 6 & 24 \\
\hline N Te Sxt Gm Fm Nfx Ls & & & & & 1 & & & 1 \\
\hline N Te Sxt Gm Fm Nfx C & 2 & 6 & 1 & & 5 & 4 & 1 & 19 \\
\hline N Te Sxt Fm Nfx C Ls & & 5 & 2 & 1 & 1 & 2 & 3 & 14 \\
\hline N Te Sxt Gm Fm C Ls & & & & & & & 1 & 1 \\
\hline N Te Sxt Gm Fm Nfx & 1 & 2 & & & & 2 & & 5 \\
\hline Te Sxt Gm Fm Nfx C & & & & & & & 1 & 1 \\
\hline N Te Sxt Gm Fm Ls & & & & & & 1 & & 1 \\
\hline N Te Sxt Fm Nfx C & 6 & & 4 & 1 & 2 & 5 & 7 & 25 \\
\hline N Te Sxt Gm Fm C & & & 1 & 1 & & & & 2 \\
\hline N Te Sxt Fm C Ls & & & 1 & & & & & 1 \\
\hline N Te Sxt Fm Nfx & 3 & 8 & & & & & 4 & 15 \\
\hline Te Fm Nfx C Ls & & & & & & & 1 & 1 \\
\hline N Te Sxt Nfx C & 1 & & & & & & & 1 \\
\hline $\mathrm{N}$ Te Sxt Fm C & & & & & & 1 & & 1 \\
\hline N Te Sxt C Ls & 1 & & & & & & & 1 \\
\hline Te Sxt Fm Nfx & & 1 & & & & & & 1 \\
\hline N Te Sxt Fm & 1 & & & 1 & & & & 2 \\
\hline N Te Sxt Gm & & & & & 1 & & & 1 \\
\hline $\mathrm{N}$ Te Fm C & 1 & & & & & & & 1 \\
\hline N Te Sxt C & & & & & & & 1 & 1 \\
\hline N Te Sxt & & & 1 & & & & & 1 \\
\hline Te Fm & 1 & & & & & & & 1 \\
\hline Total No. $\%$ & $21(17.35)$ & $29(23.96)$ & $12(9.91)$ & $7(5.78)$ & $11(9.09)$ & $16(13.22)$ & $25(20.66)$ & $121(100.00)$ \\
\hline
\end{tabular}


Ninety nine $(81.81 \%)$ isolates fell into six phylogenetic subgroups including: 21 isolates (17.35\%) $\mathrm{A}_{0}, 29$ isolates $(23.96 \%) \mathrm{A}_{1}, 7$ isolates $(5.78 \%) \mathrm{B} 2_{2}, 11$ isolates $(9.09 \%) \mathrm{B}_{2}, 16$ isolates $(13.22 \%) \mathrm{D}_{1}$ and 25 isolates $(20.66 \%) \mathrm{D}_{2}$. Phylogenetic subgroup of $\mathrm{A}_{1}$ was the most prevalent followed by $\mathrm{D}_{2}$ (Table 2).

All of the $E$. coli isolates from $\mathrm{O} 78$ serogroup belonged to A phylo-group whereas the $\mathrm{O} 2$ isolates mostly fell into group D.

All of the 121 isolates showed resistance to two or more antibiotics. The maximum resistance was against tetracycline $100 \% \quad(121$ isollates $)$ followed by trimethoprim-sulfamethxazole $(97.52 \%)$, neomycin (96.69\%), flumequine $(95.86 \%)$ and enrofloxacin $(88.42 \%)$. The minimal resistance was against lincospectin (37.19\%) then gentamicin (45.45\%) and chloramphenicol $(76.85 \%)$. Twenty three different patterns of multiple drug resistance were observed, out of which N-Te-Sxt-Fm-Nfx-C and N-Te-Sxt-Gm-Fm$\mathrm{Nfx}-\mathrm{C}$-Ls were the two most frequent patterns respectively. The resistant isolates were found in all of the phylogenetic groups and or sub groups (Table 2).

\section{DISCUSSION}

Enterobacteriaceae form a large, heterogeneous family of medically and scientifically important, gramnegative bacteria. Escherichia coli, the most prevalent infecting organism in this family, is one of the prototypic bacteria studied (Carnes and Wilkins, 2005). Coliform salpingitis/salpingoperitonitis results in decreased egg production and sporadic mortality. It is one of the most common causes of mortality in commercial layers and breeder chickens (Saif et al., 2003). In the present study, 47 isolates were typeable and belonged to 11 serogroupes. Monroy et al. (2005) have reported that $E$. coli isolates from broiler breeders with salpingitis belong to serogroups $\mathrm{O} 1, \mathrm{O} 2, \mathrm{O} 5, \mathrm{O} 36$, O45, O53 and O78. In a study on five clinically normal chickens from three farms $E$. coli isolates from the peritoneal cavity were serogrouped as O78 and 0111 (Trampel et al., 2007). Variation in O-serogroups of isolates according to geographic region occur, but in most studies the common serotypes of APEC isolates have been O1, O2, O35, and O78 (Saif et al., 2003). Isolates of APEC from birds with salpingitis have more similarity with $E$. coli isolates from airsaculitis. Although in a study on the molecular epidemiology of $E$. coli isolates from an infection outbreak in a layer breeder flock the epidemic clone was also found in an environmental sample, suggest have been transmitted to the flock via the environment (Timothy et al., 2008). The present observation indicated that $\mathrm{O} 78$ is the most prevalent serogroup in salpingitis derived isolates. This serogroup is known to include virulent strains associated with sever $E$. coli infection in poultry. It is also recognized as one of the serogroups associated with enterotoxigenic $E$. coli strains that can affect humans (Calnek et al., 1997; La Ragione and Woodward, 2002).

In this study high frequency of resistant isolates against tetracycline ascribe to its frequent use as therapeutic and feed additive. On the other hand, 24 isolates $(19.83 \%)$ were resistant to all of the examined antibiotics. Avian pathogenic E. coli are often highly resistant, especially to tetracycline, streptomycin and sulfonamides. High levels of resistance to ciprofloxacin have been reported from China. In medicine, frequency of strains resistant to ampicillin, trimethoprimsulfamethoxazole, streptomycin, Ciproflaxin, chloramphenicol, tetracycline and cephalosporins (ceftriaxone or ceftazidime) is a cause of growing concern (Raja et al., 2009). Commensal E. coli strains from poultry have similar patterns of resistance but at lower frequencies (Gyles, 2008). A study on prevalence and distribution of antiobiotic resistance in $E$. coli isolates from pigs and poultry showed that the isolates had high rates of resistance to ampicillin (99.5\%), doxycycline (95.6\%), tetracycline (93.4\%), trimethoprim-sulfamethoxazole $(74.3 \%)$, amoxicillin $(65.1 \%)$, streptomycin $(54.7 \%)$ and chloramphenicol $(50.2 \%)$. Resistance to cephalosporins, quinolones and aminoglycosides was also quite prevalent ( $\mathrm{Li}$ et al., 2010). Antibiotic administration is considered the most important factor promoting the emergence, selection and dissemination of antibiotic-resistant microorganisms in both veterinary and human medicine. Antibiotic usage selects for resistance not only in pathogenic bacteria but also in the endogenous flora of exposed individuals (animals and humans) or populations (Van den Bogaard et al., 2001). Diarrassouba et al. (2007) indicated that multiple antibiotic-resistant commensal E. coli and Salmonella strains carrying virulence genes can be found on commercial broiler chicken farms and may provide a reservoir for these genes in chicken production facilities. Recent study provides clear evidence that a sequential medication of a broiler flock, with different antimicrobial classes during short periods of time for prophylactic objectives, was accompanied by a dramatic increase in both antimicrobial resistance rates and phenotype diversity of E. coli strains (Da Costa et al., 2008). Development of resistant pathogenic E. coli strains pose considerable threat to public health as these strains after ingestion, are capable of transferring their resistance to other pathogens through episomal transfer of R-factor 
(Johnson et al., 2008). In human derived isolates, the most common resistance mechanism in Gram-negative bacteria includes production of -lactamases that hydrolyze the antibiotic ring, thus deactivating antimicrobial activity (Kathryn et al., 2005).

There are few reports on phylogentic background of salpingitis associated $E$. coli isolates. In the current study, salpingitis derived $E$. coli isolates segregated in phylogenetic groups A (41.32\%), D (33.88\%), B2 (14.87\%) and B1 (9.91\%). Trampel et al. (2007) reported that $E$. coli isolates from peritonitis lesions in commercial laying hens belonged to $\mathrm{A}$ and $\mathrm{D}$ phylogenetic groups. According to virulence genotype and phylogenetic group $E$. coli isolates from the magnum and peritoneum are similar to APEC isolates. Dissanayake et al. (2008) showed that APEC isolates belong to A $(71 \%), \mathrm{B} 1(4.1 \%), \mathrm{B} 2(7.9 \%)$ and $\mathrm{D}$ (18.65\%) groups. Phylogenetic analyses of E. coli isolates from avian cellulitis showed that $E$. coli isolates belonged to A $(51.56 \%)$, B1 (18.75\%) and D (29.68\%) phylogenetic groups (Ghanbarpour et al., 2009). Rodriguez-Siek et al. (2005) found that APEC strains fell into A (38.0\%), D (28.1\%), B2 (18.5\%) and B1 (15.5\%) phylo-groups. Results of phylo-typing showed that considerable portion of APEC strains also fell into group a $(46.1 \%)$ and B2 $(35.1 \%)$ (Ewers et al., 2009). According to the results of this study multi antibiotic resistant, isolates were found in four phylogenetic groups. Johnson et al. (2003) reported that nalidixic acid-resistant $E$. coli isolates from retail chicken products belonged to phylo groups B2 and D. The findings of a study on antimicrobial resistance profiles among $E$. coli isolates from human and animal wastewater led to hypothesize that abusive antibiotic use in food animal production may promote the development of resistance among the intestinal E. coli phylogroups (Sabate et al., 2008).

\section{CONCLUSION}

In conclusion, comparison of the results of the current study with finding of other reports revealed that there are similarities in phylogroups, O-serogroups and antibio-resistance profiles between salpingitis derived $E$. coli strains and other APEC isolates. It is imperative to determine antibiotic sensitivity of $E$. coli isolates involved in different disease so that ineffective antibiotics can be avoided.

\section{ACKNOWLEDGMENT}

This study was supported by a grant from Shahid Bahonar University of Kerman. The authors are thankful to Dr. Eric Oswald (Ecole National Veterinaire Toulouse, France) for providing the reference strains.

\section{REFERENCES}

Calnek, B.W., H.J. Barnes, C.W. Beard, L.R. Mcdougald and Y.M. Saif, 1997. Diseases of Poultry. 10th Edn., Iowa State Press, London, ISBN: 0813804272, pp: 1080.

Carnes, E. and E. Wilkins, 2005. The development of a new, rapid, amperometric immunosensor for the detection of low concentrations of bacteria part II: optimization of the system for Escherichia coli. Am. J. Applied Sci., 2: 607-613. http://www.scipub.org/fulltext/ajas/ajas23607-613.pdf

Clermont, O., S. Bonacorsi and E. Bingen, 2000. Rapid and simple determination of the Escherichia coli phylogenetic group. Applied Environ. Microbiol., 66: 4555-4558. PMID: 19474064

Da Costa, P.M., A. Bica, P. Vaz-Pires and F. Bernardo, 2008. Effects of antimicrobial treatment on selection of resistant Escherichia coli in broiler fecal flora. Microb. Drug. Resist., 14: 299-306. PMID: 19025467

Diarrassouba, F., M.S. Diarra, S. Bach, P. Delaaquis and J. Prtahard et al., 2007. Antibiotic resistance and virulence genes in commensal Escherichia coli and Salmonella isolates from commercial broiler chicken farms. J. Food. Prot., 70: 1316-1327. PMID: 17612058

Dissanayake, D.R., T.G. Wijewardana, G.A. Gunawardena and I.R. Poxton, 2008. Distribution of lipopolysaccharide core types among avian pathogenic Escherichia coli in relation to the major phylogenetic groups. Vet. Microbiol., 132: 355-363. PMID: 18597955

Escobar-Paramo, P., A. Le Menac'h, T. Le Gall, C. Amorin and S. Gouriou et al., 2006. Identification of forces shaping the commensal Escherichia coli genetic structure by comparing animal and human isolates. Environ. Microbiol., 8: 1975-1984. PMID: 17014496

Escobar-Paramo, P., O. Clermont, A.B. Blanc-Potard, H. Bui and C. Le, Bouguenec et al., 2004a. A specific genetic background is required for acquisition and expression of virulence factors in Escherichia coli. Mol. Biol. Evol., 21: 1085-1094. PMID: 15014151

Escobar-Paramo, P., K. Grenet, A. Le Menac'h, L. Rode and E. Salgado et al., 2004b. Large-scale population structure of human commensal Escherichia coli isolates. Applied Environ. Microbiol., 70: 5698-5700. PMID: 15345464 
Ewers, C., E.M. Antao, I. Diehl, H.C. Philipp and L.H. Wieler, 2009. Intestine and environment of the chicken as reservoirs for extraintestinal pathogenic Escherichia coli strains with zoonotic potential. Applied Environ. Microbiol., 75: 184-192. PMID: 18997030

Fossum, O., D.S. Jansson, P.E. Etterlin and I. Vagsholm, 2009. Causes of mortality in laying hens in different housing systems in 2001 to 2004. Acta Vet. Scand., 15: 51-53. DOI: 10.1186/17510147-51-3

Germon, P., Y.H. Chen, L. He, J.E., Blanco and A. Bree et al., 2005. ibeA, a virulence factor of avian pathogenic Escherichia coli. Microbiology, 151: 1179-86. PMID: 15817785

Ghanbarpour, R. and M. Salehi, 2010. Determination of adhesin encoding genes in Escherichia coli isolates from omphalitis of chicks. Am. J. Anim. Vet. Sci., 5: 91-96. http://www.scipub.org/fulltext/AJAV/AJAV5291-96

Ghanbarpour, R., M. Salehi and E. Oswald, 2009. Virulence genotyping of Escherichia coli isolates from avian cellulites in relation to phylogeny. Comp. Clin. Pathol., 19: 147-153. DOI: 10.1007/s00580-009-0837-4

Gordon, D.M., O. Clermont, H. Tolley and E. Denamur, 2008. Assigning Escherichia coli strains to phylogenetic groups: Multi-locus sequence typing versus the PCR triplex method. Environ. Microbiol., 10: 2484-2496. PMID: 18518895

Gordon, D.M., S.E. Stern and P.J. Collignon, 2005. The influence of the age and sex of human hosts on the distribution of Escherichia coli ECOR groups and virulence traits. Microbiology, 151: 15-23. PMID: 15632421

Gyles, C., 2008. Veterinary medicine and public health. Can Vet J., 24: 1163-1164. PMID: 19252708

Johnson, J.R., A.C. Murray, A. Gajewski, M. Sullivan and P. Snippes et al., 2003. Isolation and molecular characterization of nalidixic acid-resistant extraintestinal pathogenic Escherichia coli from retail chicken products. J. Antimicrob. Chemother., 47: 2161-2168. PMID: 12821463

Johnson, T.J., Y. Wannemuehler, S.J. Johnson, A.L. Stell and C. Doetkott et al., 2008. Comparison of extraintestinal pathogenic Escherichia coli strains from human and avian sources reveals a mixed subset representing potential zoonotic pathogens. Applied Environ. Microbiol., 74: 7043-7050. PMID: 18820066

Jordan, F.T., N.J. Williams, A. Wattert and T. Jones, 2005. Observations on salpingitis, peritonitis and salpingoperitonitis in a layer breeder flock. Vet. Rec., 5: 573-577. PMID: 16272543
Jorgensen, J.H. and J.F. Hindler, 2007. New consensus guidelines from the clinical and laboratory standards institute for antimicrobial susceptibility testing of infrequently isolated or fastidious bacteria. Med. Microbiol. 44: 280-286. PMID: 17173232

Kathryn, J.E., J.L. Kuti and D.P. Nicolau, 2005. Pharmacodynamic comparison of the Carbapenems against E. coli and Klebsiella spp. containing extended spectrum-lactamases. Am. J. Infect. $\quad$ Dis., 1 : 149-155. http:/www.scipub.org/fulltext/ajid/ajid13149-155.pdf

La Ragione, R.M. and M.J. Woodward, 2002. Virulence factors of Escherichia coli serotypes associated with avian colisepticaemia. Res. Vet. Sci., 73: 27-35. PMID: 12208104

Landman, W.J. and R.A. Cornelissen, 2006. Escherichia coli salpingitis and peritonitis in layer chicken overview. Tijdschr. Diergeneeskd, 15: 814-822. PMID: 17263015

Li, L., Z.G. Jiang, L.N. Xia, J.Z. Shen and L. Dai et al., 2010. Characterization of antimicrobial resistance and molecular determinants of beta-lactamase in Escherichia coli isolated from chickens in China during 1970-2007. Vet. Microbiol., 144: 505-510. PMID: 20207509

Monroy, M.A., T. Knobl, J.A. Bottino, C.S. Ferreira and A.J. Ferreira, 2005. Virulence characteristics of Escherichia coli isolates obtained from broiler breeders with salpingitis. Comp. Immunol. Microbiol. Infect. Dis., 28: 1-15. PMID: 15563950

Neubauer, C., M. de Souza-Pilz, A.M. Bojesen, M. Bisgaard and M. Hess, 2009. Tissue distribution of haemolytic Gallibacterium anatis in laying birds with reproductive disorders. Avian. Pathol., 38: 1-7. PMID: 19089694

Pattison, M., P.F. McMulin, J.M. Brabury and D. Alexander, 2007. Poultry Disease. 6th Edn., Saunders Ltd., London, ISBN: 0702028622, pp: 632.

Raja, S.B., M.R. Murali, G.K. Malathi, K. Anbarasu and S.N. Devaraj, 2009. Effect of aqueous extract of aegle marmelos fruit on adherence and $\beta$-lactam resistance of enteropathogenic Escherichia coli by down regulating outer membrane Protein C. Am. J. Infect. Dis., 5: 154-162. http://www.scipub.org/fulltext/ajid/ajid52154-162.pdf

Rodriguez-Siek, K.E., C. W. Giddings, C. Doetkott, T.J. Johnson and L.K. Nolan, 2005. Characterizing the APEC pathotype. Vet. Res., 36: 241-256. PMID: 15720976

Sabate, M., G. Prats, E. Moreno, E. Balleste and A.R. Blanch et al., 2008. Virulence and antimicrobial resistance profiles among Escherichia coli strains isolated from human and animal wastewater. Res. Microbiol., 159: 288-293. PMID: 18434099 
Saif, Y.M., A.M. Fadly, J.R. Glisson, L.R. McDougald and L.K. Nolan et al., 2003. Diseases of Poultry. 11th Edn., Wiley-Blackwell, London, ISBN: 9780813804231, pp: 1260.

Soliman, E.S. E. Taha, K.D. Infante, K. Laboy, M.A. Sobieh and P.G. Reddy, 2009. Stressors influence on Salmonella enterica Serovar Enteritidis colonization in broilers. Am. J. Anim. Vet. Sci., 4: 42-48.

http://www.scipub.org/fulltext/AJAV/AJAV434248.pdf

Timothy, S., K. Shafi, A.H. Leatherbarrow, F.T. Jordan and P. Wigley, 2008. Molecular epidemiology of are productive tract associated colibacillosis outbreak in a layer breeder flock associated with atypical avian pathogenic Escherichia coli. Avian. Pathol., 37: 375-378. PMID: 18622852
Trampel, D.W., Y. Wannemuehler and L.K. Nolan, 2007. Characterization of Escherichia coli isolates from peritonitis lesions in commercial laying hens. Avian. Dis., 51: 840-844. PMID: 18251391

Van den Bogaard, A.E., N. London, C. Driessen and E.E. Stobbering, 2001. Antibiotic resistance of faecal Escherichia coli in poultry, poultry farmers and poultry slaughterers. J. Antimicrob. Chemother., 47: 763-771. PMID: 11389108

Walk, S.T., E.W. Alm, L.M. Calhoun, J.M. Mladonicky and T.S. Whittman, 2007. Genetic diversity and population structure of Escherichia coli isolated from fresh water beaches. Environ. Microbiol., 9: 2274-2288. PMID: 17686024 